This item was submitted to Loughborough's Institutional Repository (https://dspace.lboro.ac.uk/) by the author and is made available under the following Creative Commons Licence conditions.

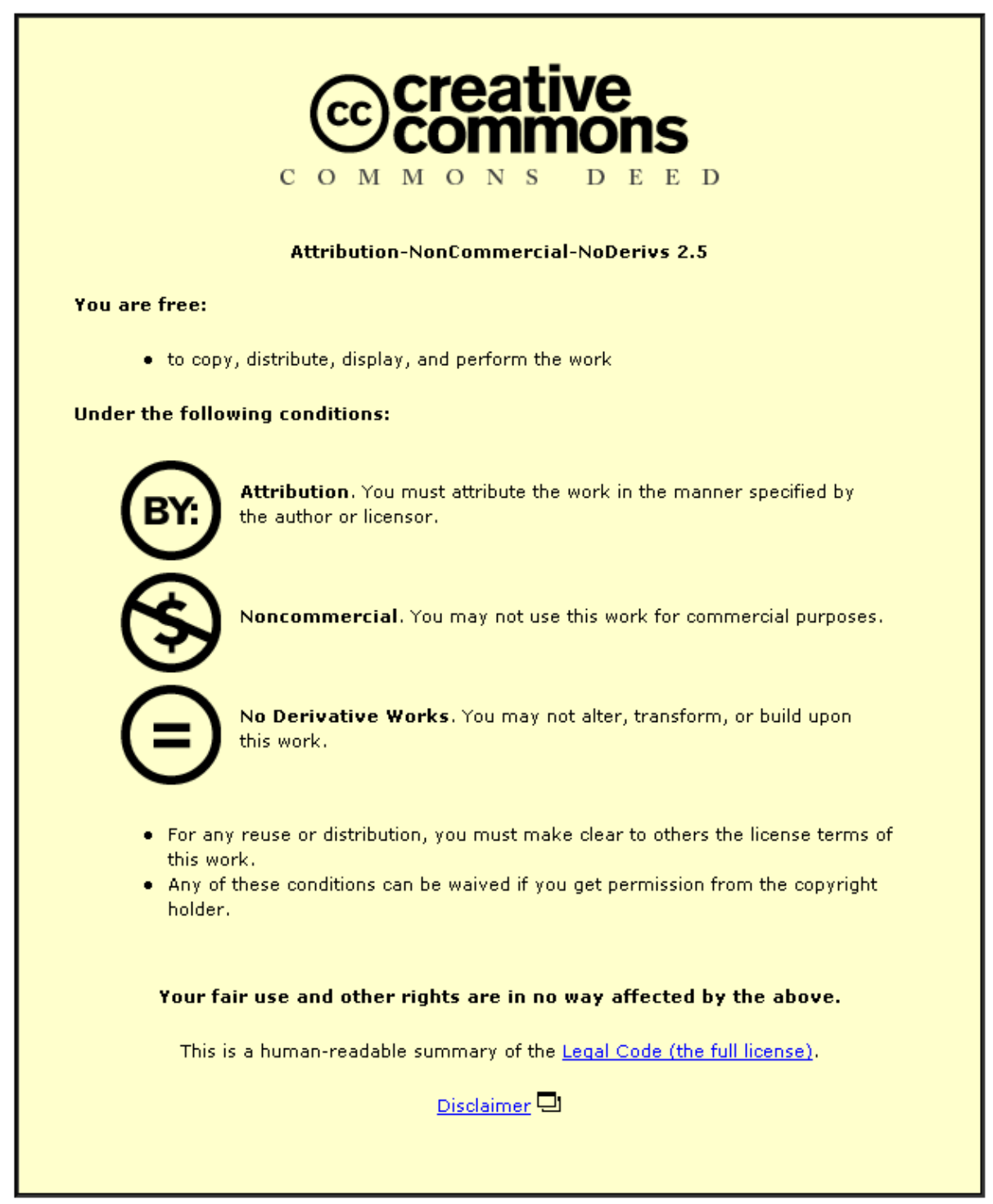

For the full text of this licence, please go to: http://creativecommons.org/licenses/by-nc-nd/2.5/ 


\title{
Trapping of lattice polarons by impurities
}

\author{
J. P. Hague, ${ }^{1,2}$ P. E. Kornilovitch, ${ }^{3}$ and A. S. Alexandrov ${ }^{1}$ \\ ${ }^{1}$ Department of Physics, Loughborough University, Loughborough, LE11 3TU, United Kingdom \\ ${ }^{2}$ Department of Physics and Astronomy, The Open University, Milton Keynes, MK7 6AA, United Kingdom \\ ${ }^{3}$ Hewlett-Packard Company, 1000 NE Circle Blvd, Corvallis, Oregon 97330, USA
}

(Received 16 May 2008; published 16 September 2008)

\begin{abstract}
We consider the effects of impurities on polarons in three dimensions using a continuous time quantum Monte Carlo algorithm. An exact treatment of the phonon degrees of freedom leads to a very efficient algorithm, and we are able to compute the polaron dynamics on an infinite lattice by developing an auxiliary weighting scheme. The magnitude of the impurity potential, the electron-phonon coupling, and the phonon frequency are varied. We determine the magnitude of the impurity potential required for polaron trapping. For small electron-phonon coupling the number of phonons increases dramatically on trapping combined with a sharp decrease in kinetic energy. The polaron binding diagram is computed, showing that intermediatecoupling low-phonon-frequency polarons are localized by exceptionally small impurities.
\end{abstract}

DOI: 10.1103/PhysRevB.78.092302

PACS number(s): $71.38 .-\mathrm{k}$

Interest in the role of electron-phonon interactions (EPIs) and polaron dynamics has recently gone through a vigorous revival. Polarons have been shown to be relevant in hightemperature superconductors, colossal magnetoresistance oxides, polymers, and many inorganic semiconductors; electron transport through nanowires often depends on vibronic displacements of ions. The continued interest in polarons extends well beyond the physical description of advanced materials. The field has been a testing ground for analytical, semianalytical, and different numerical techniques. ${ }^{1}$

The situation as regards to polaron formation and dynamics in real materials is complicated by an intrinsic disorder. Moreover, the EPI cannot be considered either weak or strong in many of the materials described above, so standard approximations based on perturbation theories break down. In a pioneering paper Economou and coauthors ${ }^{2}$ studied a one-dimensional (1D) large polaron with diagonal disorder by using methods from the theory of nonlinear systems. Bronold et al. ${ }^{3}$ investigated the dynamics of a single electron in a Holstein model with a site-diagonal, binary-alloy-type disorder by applying a dynamical mean-field theory (DMFT) for a Bethe lattice with infinite coordination number. DMFT was further improved by Bronold and Fehske, ${ }^{4}$ who described Anderson localization and the self-trapping phenomena within the same model.

There is a delicate interplay between the self-trapping by EPI and trapping by dopant-induced disorder in the intermediate-coupling regime, so even a single polaron has to be studied by exact methods such as continuous-time quantum Monte Carlo (CTQMC), which was developed for the free lattice polaron and bipolaron in Refs. 5 and 6 , respectively, and/or diagrammatic Monte Carlo (DMC) ${ }^{7}$ techniques. In particular, the CTQMC algorithm treats the phonon degrees of freedom exactly, it does not suffer from time discretization errors, and it is not limited to finite lattices, thus providing numerically exact solution of the (bi)polaron problem in any dimensions and on any lattice. While the properties of free (bi)polarons are very important, the interplay between polarons and disorder should also be addressed to understand the properties of many materials.

To address this crucial question, we make a further devel- opment of the CTQMC algorithm, so that the algorithm can be used to solve the problem of the electron interacting with phonons in the presence of an impurity on a threedimensional (3D) lattice with no finite size errors. The electron hopping is assumed to be between nearest neighbors only. The phonon subsystem is made up of independent oscillators with frequency $\omega$, displacement $\xi_{\mathrm{m}}$, momentum $\hat{P}_{\mathbf{m}}=-i \hbar \partial / \partial \xi_{\mathbf{m}}$, and mass $M$ associated with each lattice site. The real space Hamiltonian reads

$$
\begin{aligned}
H= & -t \sum_{\left\langle\mathbf{n n}^{\prime}\right\rangle} c_{\mathbf{n}^{\prime}}^{\dagger} c_{\mathbf{n}}+\sum_{\mathbf{n}} \Delta_{\mathbf{n}} c_{\mathbf{n}}^{\dagger} c_{\mathbf{n}}+\sum_{\mathbf{m}}\left(\frac{\hat{P}_{\mathbf{m}}^{2}}{2 M}+\frac{M \omega^{2} \xi_{\mathbf{m}}^{2}}{2}\right) \\
& -\sum_{\mathbf{n} \mathbf{m}} f_{\mathbf{m}}(\mathbf{n}) c_{\mathbf{n}}^{\dagger} c_{\mathbf{n}} \xi_{\mathbf{m}} .
\end{aligned}
$$

Here $\left\langle\mathbf{n n}^{\prime}\right\rangle$ denote pairs of nearest neighbors. Sites are indexed by $\mathbf{n}$ for electrons and $\mathbf{m}$ for ions. Spin indices and Hubbard $U$ are omitted since there is only one electron. The strength of the EPI is defined through a dimensionless coupling constant, $\lambda=\Sigma_{\mathbf{m}} f_{\mathbf{m}}^{2}(0) / 2 M \omega^{2} z t$, which is the ratio of the polaron energy when $t=0$ to the kinetic energy of the free electron $W=z t$. In this paper, we discuss Holstein polarons, which have a force function, $f_{\mathbf{m}}(\mathbf{n})=\kappa \delta_{\mathbf{n}, m} \cdot{ }^{8}$ A special case of the Hamiltonian given in Eq. (1) is the case where the external potential, $\Delta_{\mathbf{n}}=\Delta \delta_{\mathbf{n}, 0}$, represents an impurity. We will study this in detail in this paper.

The main development here is the extension of the CTQMC algorithm for polaron trapping by an impurity. The effective polaron action that results when the phonon degrees of freedom have been integrated out analytically is given by

$$
\begin{aligned}
A[\mathbf{r}(\tau)]= & \frac{z \lambda \bar{\omega}}{2} \int_{0}^{\bar{\beta}} \int_{0}^{\bar{\beta}} d \bar{\tau} d \bar{\tau}^{\prime} e^{-\bar{\omega} \bar{\beta} / 2} \cosh \left[\bar{\omega}\left(\bar{\beta} / 2-\left|\bar{\tau}-\bar{\tau}^{\prime}\right|\right)\right] \\
& \times \frac{\Phi_{0}\left[\mathbf{r}(\tau), \mathbf{r}\left(\tau^{\prime}\right)\right]}{\Phi_{0}(0,0)}-\int_{0}^{\beta} \Delta[\mathbf{r}(\tau)] d \tau
\end{aligned}
$$

The contribution of this action to the statistical weight of a path configuration is $e^{A}$. $\Phi_{0}\left[\mathbf{r}(\tau), \mathbf{r}\left(\tau^{\prime}\right)\right]$ 
$=\sum_{\mathbf{m}} \bar{f}_{\mathbf{m}}[\mathbf{r}(\tau)] \bar{f}_{\mathbf{m}}\left[\mathbf{r}\left(\tau^{\prime}\right)\right]$ is the phonon mediated selfinteraction (here $\bar{\omega}=\omega / t$ and $\bar{\beta}=t / k_{B} T$ ).

One of the main complications regarding the simulation of a particle in a single impurity potential is ensuring that the whole configuration space is sampled. This can be understood in the following way. During a binary kink update (discussed below) the ends of the path may either stay put or move along one of the nearest-neighbor bonds, i.e., the path is essentially a random walker. In a thought experiment, we set up the system with a very small attractive $\Delta \rightarrow 0^{-}$, with the path close to the impurity. In $1 \mathrm{D}$, the random walker has finite probability to return to the start site after a finite number of steps. In two dimensions (2D) the walker has vanishing probability to return, so ergodicity is not guaranteed. In $3 \mathrm{D}$, the walker will not in general return to its start point even after an infinite time, so if updates only have the properties of a basic random walker, then the Monte Carlo procedure will fail, since the impurity will (on average) never be visited.

In order to ensure that the path includes sufficient sampling of the impurity, it would be useful to make the path spend more time near the impurity, weighting the estimators in such a way that the final measurements are the same. Such sampling may be achieved by introducing an auxiliary weighting to the problem. A functional of the path $w[\{C\}]$ is introduced, which represents the probability that an unbound path is found in a particular configuration. $w[\{C\}]$ is tuned so that the path samples the impurity regularly, modifying the measurements as

$$
\begin{aligned}
\langle O\rangle=\langle\hat{O}\rangle_{A_{\mathrm{tot}}} & =\frac{\sum_{\{C\}} O[\{C\}] e^{A_{\mathrm{tot}}[\{C\}]}}{\sum_{\{C\}} e^{A_{\mathrm{tot}}[\{C\}]},} \\
& =\frac{\sum_{\{C\}} O[\{C\}] w[\{C\}] e^{A_{\mathrm{tot}}[\{C\}] / w[\{C\}]}}{\sum_{\{C\}} w[\{C\}] e^{A_{\mathrm{tot}}[\{C\}] / w[\{C\}]},} \\
& =\frac{\langle\hat{O} / w[\{C\}]\rangle_{A_{\mathrm{tot}}, w}}{\langle 1 / w[\{C\}]\rangle_{A_{\mathrm{tot}}, w}}
\end{aligned}
$$

and the update probabilities are modified by the ratio of weights $w[\{D\}] / w[\{C\}]$ on changing from configuration $\{C\}$ to $\{D\} . A_{\text {tot }}$ is the total action including kinetic energy terms (for reasons relating to the continuous time algorithm, the kinetic energy part of the statistical weight is taken into account through the update rule below). Thus the measurement of an observable with respect to the ensemble defined by the action $A_{\text {tot }}$ can be related to the measurement of an observable with respect to the ensemble defined by $A_{\text {tot }}$ and $w$. Error bar estimation is an important aspect of Monte Carlo simulation, for which we use the bootstrap method. It is necessary to take into account the covariance between $\langle\hat{O} / w[\{C\}]\rangle_{A_{\mathrm{tot}}, w}$ and $\langle 1 / w[\{C\}]\rangle_{A_{\mathrm{tot}}, w}$ when determining error bars.

In the weighted ensemble, the ground-state polaron energy, $E$, the kinetic energy, and number of phonons are com- puted using the estimators in, e.g., Ref. 9 modified according to Eqs. (3)-(5). The average distance from the impurity is computed via

$$
R_{\mathrm{imp}}=\left\langle\frac{1}{w} \sqrt{\frac{1}{\beta} \int_{0}^{\beta} \mathbf{r}^{2}(\tau) d \tau}\right\rangle_{A_{\mathrm{tot}}, w} /\left\langle\frac{1}{w}\right\rangle_{A_{\mathrm{tot}}, w} .
$$

The form of $w$ is a matter of choice, but it is useful to choose a form that allows control over the confinement of the new path. To avoid undesirable long timestep correlations, we use an auxiliary weighting of the form $w \propto 1 /(\alpha$ $\left.+R^{d+1+\eta}\right)$, where $R$ is the distance of the configuration from the impurity, $d$ the dimensionality of the lattice, $\eta$ is a small value, and $\alpha$ stops the weight blowing up on the impurity site. In this way, the average distance from the impurity is finite since in the absence of interaction, $\langle R\rangle$ $\sim \int_{0}^{\infty} w[R] R^{d-1} d R$ shows that "free" particles are localized, but not bound too strongly. We found $\alpha=10, \eta=0.1$ to be a good compromise in both bound and unbound cases.

In the path integral quantum Monte Carlo (QMC) for a polaron in an impurity, it is necessary to make update operations involving two kinks to ensure that the end configurations of the path remain periodic in imaginary time, since the translational symmetry has been broken. A binary update satisfying the imaginary-time boundary conditions involves adding or removing a kink-antikink pair (an antikink to kink $\mathbf{1}$ is a kink with direction -1). Update probability is determined following a similar argument to that in Ref. 9 with a small modification; consider two path configurations, $\{C\}$ and $\{D\}$, where configuration $\{D\}$ has one more 1 kink at time $\tau_{1}$ and one more $-\mathbf{l}$ antikink at time $\tau_{2}$ than $\{C\}$. The balance equation is $W[\{C\}] Q_{A}[\{C\}] P[\{C\} \rightarrow\{D\}]$ $=W[\{D\}] Q_{R}[\{D\}] P[\{D\} \rightarrow\{C\}]$. With relative contribution of configurations $\{C\}$ and $\{D\}$ modified by the auxiliary weighting, $W[\{D\}] / W[\{C\}]=(t \Delta \tau)^{2} e^{A[\{D\}]-A[\{C\}]} w[\{D\}] / w[\{C\}]$.

We modify the equal weighting scheme in Ref. 9 for the probability of choosing kinks with a particular kink time when adding or removing the second kink, allowing weighted kink insertion with the antikink time chosen with probability $p\left(\tau-\tau^{\prime}\right)$, where $\int_{0}^{\beta} p(\tau) d \tau=1$. In this way, kinks and antikinks in the pair can be chosen at similar $\tau$, and the update acceptance is improved. This choice leads to update probabilities for insertion of a direction $\mathbf{1}$ kink at $\tau$ and a direction $-\mathbf{l}$ kink at $\tau^{\prime}, \quad P[C \rightarrow D]$ $=\min \left\{1 ; w[D] t_{1} t_{-1} \beta e^{A[D]-A[C]} / w[C] N_{\mathrm{l}}[D] \sum_{i=1}^{N_{-1}[D]} p\left(\tau, \tau_{i}\right)\right\}$

In order to sample the configuration space faster, we also examined path updates which shift the whole path through a certain distance, but the efficiency of the algorithm was not improved. The auxiliary weighting approach may be used for systems with several particles, with the auxiliary weight depending on either the absolute or relative positions of the paths.

Applying the Lang-Firsov (LF) canonical transformation to the Hamiltonian and assuming large phonon frequency, an approximate form can be derived, $\widetilde{H}=-\widetilde{t} \Sigma_{\langle i j\rangle} c_{i}^{\dagger} c_{j}+\Sigma_{i} \Delta_{i} c_{i}^{\dagger} c_{i}$, with $\tilde{t}=t \exp (-z t \lambda / \omega)$. It is possible to compute an analytic value of the impurity potential, $\Delta$ at which binding occurs. For polarons on a 3D lattice under the influence of an impurity, $\Delta_{C}=3.958 \tilde{t}=3.958 t m_{0} / m^{*}$, where $m_{0} \propto 1 / t$ is the band 


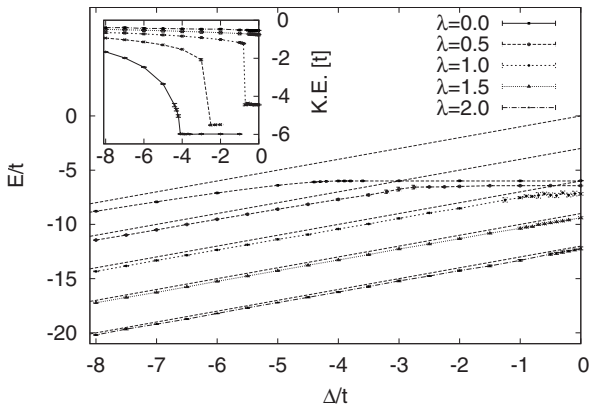

FIG. 1. Total energy $E$ vs $\Delta$ for various $\lambda$ at $\bar{\omega}=1$. In all figures, error bars correspond to three standard errors. Where error bars are not visible, error bars are smaller than the points. Straight dotted lines indicate the strong impurity asymptote $-z t \lambda+\Delta$. Inset: Kinetic energy.

mass in a rigid lattice and $m^{*} \propto 1 / \tilde{t}$ is the polaron mass. When there is no electron-phonon coupling, the relation $\Delta_{C}$ $=3.958 t$ is exact. While the approximation for $\tilde{t}$ is not expected to hold for low phonon frequency, use of the exact value of the inverse mass computed from CTQMC in the absence of the impurity is expected to lead to a qualitatively correct value for $\Delta_{C}$. We will return to this point later in the article. An exact value for the energy of the instantaneous problem is given by the equation, $1=|\Delta| \iiint_{-\pi}^{\pi} d^{3} \mathbf{q} /(2 \pi)^{3} 1 /\left[|E|-2 t \sum_{i=1}^{d} \cos \left(q_{i}\right)\right]$.

To establish the circumstances under which the polaron is localized by the impurity, the total energy must be determined. Figure 1 shows the total energy $E$ vs $\Delta$ for various $\lambda$ at $\bar{\omega}=1$. The transition can be seen as a sudden change in the gradient. The flat gradient corresponds to the energy of the unbound polaron. The strong binding asymptotes show how the small polaron with large $\lambda$ almost immediately binds strongly as an impurity potential is introduced. We show the exact energy as a line beneath the $\lambda=0$ points, but for the other cases, lines are a guide to the eye. It is also interesting to see how the kinetic energy changes as the polaron binds. For weak electron-phonon coupling, the kinetic energy collapses at the transition, whereas at strong coupling the polaron is already small, and there is no significant sudden change in the kinetic energy on binding, rather a slow decrease. The $\omega \rightarrow \infty$ theory determined after taking the LF transformation does not properly show this effect; rather the $\lambda=0$ curve is just scaled and always has a kink on localization. This is evidence that exact numerics are required to properly understand the problem.

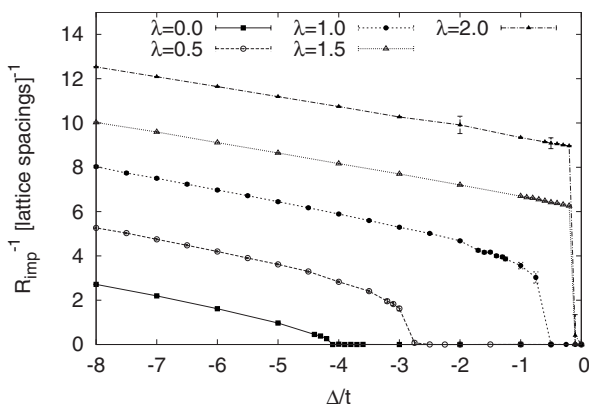

FIG. 2. $R_{\text {imp }}^{-1}$ vs $\Delta$ for various $\lambda$ at $\bar{\omega}=1$.

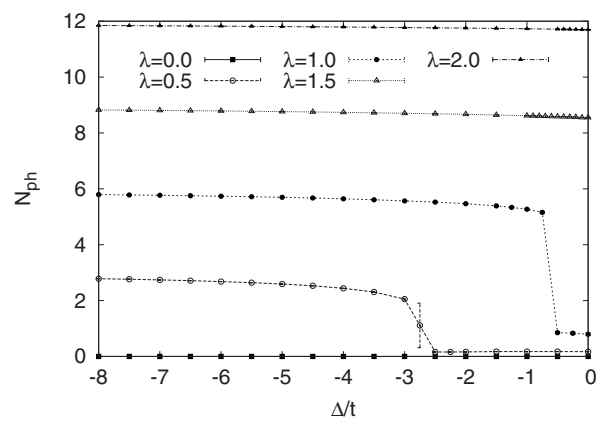

FIG. 3. $N_{p h}$ vs $\Delta$ for various $\lambda$ at $\bar{\omega}=1$. For small $\lambda$, there is a sudden increase in $N_{p h}$ as the large polaron is trapped by the impurity, leading to small polaron behavior.

The measure of the inverse radius gives another criterion for the critical binding potential. Figure 2 shows $R_{\text {imp }}^{-1}$ vs $\Delta$ for various $\lambda$ at $\bar{\omega}=1$. The inverse radius vanishes continuously on unbinding. As the electron-phonon interaction increases in strength, the polaron binds at monotonically smaller values of the impurity potential, until around $\lambda=1$, where tiny impurities are capable of binding the polaron. This is because the kinetic energy of the polaron decreases rapidly on increasing $\lambda$. Binding occurs when the depth of impurity potential is approximately the kinetic energy of the free polaron.

Small (strong-coupling) polarons contain much larger numbers of phonons than large (weak-coupling) polarons. It
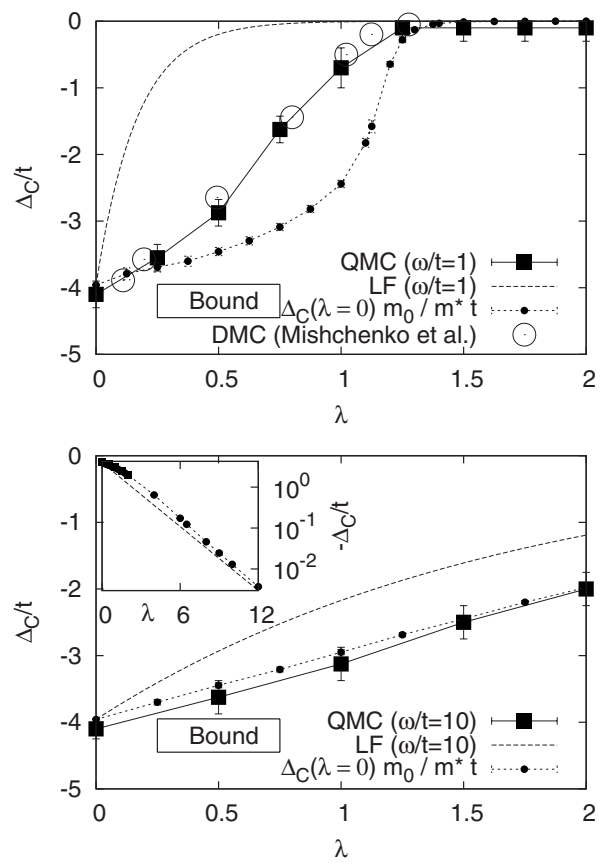

FIG. 4. Binding diagram in $\lambda, \Delta$ space. Also shown are the approximate values predicted by the LF transformation with zero excited phonons $\Delta_{C}(\lambda)=\Delta_{C}(0) \exp (-z \lambda / \bar{\omega})$, from the exact mass in the case with translational invariance (measured using QMC) $\Delta_{C}(\lambda)=\Delta_{C}(0) m_{0} / m^{*}$ and the value measured using Monte Carlo. Data obtained independently from the diagrammatic Monte Carlo approach by Mishchenko et al. (Ref. 10) are shown to agree with our method. The inset shows the convergence of $\Delta_{c}$ with the LF result for much larger $\lambda$ 
is therefore of interest to see if the reduction in size associated with localization is also associated with a similar phenomenon. Figure 3 shows the variation of $N_{p h}$ with $\Delta$ at a number of different $\lambda$ at $\omega / t=1$. For small $\lambda$, there is a sudden increase in the number of phonons as the large polaron is trapped by the impurity, consistent with the change to small polaron behavior. The localization of the polaron wave function increases the possibility for interaction between the electron and the local ion mode through a local coupling. Such an effect is expected to be less pronounced for long-range electron-phonon coupling.

Our final result is the binding diagram in $\lambda, \Delta$ space shown in Fig. 4. Also plotted are approximate values predicted by the LF transformation with zero excited phonons $\Delta_{C}(\lambda)=\Delta_{C}(0) \exp (-z \lambda / \bar{\omega})$, from the exact mass in the translationally invariant case (measured using QMC) $\Delta_{C}(\lambda)$ $=\Delta_{C}(0) m_{0} / m^{*}$ and the value measured from the current Monte Carlo code. Also shown are data obtained later and independently using DMC by Mishchenko et al. in Ref. 10. For $\bar{\omega}=1$, we examined the energy for $\bar{\beta}=112$ close to binding. Below $\bar{\beta}=56$, error bars dominated temperature corrections, and led to small differences between analytics and numerics when $\bar{\omega}=10$. At low phonon frequencies, the Holstein polaron binds almost immediately at $\lambda \gtrsim 1$. This leads to the expectation that polarons with local interaction are almost completely localized in materials with strong electronphonon coupling. Mobile behavior could re-emerge if the electron density is similar to the density of impurities and a strong Coulomb repulsion $(U \gtrsim 4 \lambda)$ is also available so that polarons become repulsive rather than attractive impurities when pinned. Longer range interactions lead to more mobile polarons, which are less likely to be pinned (the role of mass is shown by the similarity between the binding diagram from QMC, and the approximate one determined from the mass of the unbound polaron). Such long-range electron-phonon interactions are difficult to justify in 3D materials, and we expect that polarons strongly coupled with low-frequency phonons are strongly localized in such materials.

In summary, we have developed an algorithm for studying the trapping of polarons by impurities. Analysis showed that for electron-phonon coupling $\lambda>1$, low-frequency polarons are strongly bound to the impurity. For small $\lambda$, there is a more gradual binding, coupled with a sudden increase in the number of phonons present in the polaron, and sudden decrease in the kinetic energy. We computed the binding diagram, showing that critical impurity strength changes dramatically with $\lambda$ for intermediate coupling, in contrast to the conclusion for continuum (weak-coupling) polarons, and differs significantly from the strong-coupling approximation.

This work was supported by EPSRC (U.K.) (Grant No. EP/C518365/1). We thank Andrei Mishchenko and John Samson for valuable discussions.
${ }^{1}$ For recent reviews see Polarons in Advanced Materials, Springer Series in Material Sciences, edited by A. S. Alexandrov (Springer, New York, 2007).

${ }^{2}$ E. N. Economou, O. Yanovitskii, and T. Fraggis, Phys. Rev. B 47, 740 (1993)

${ }^{3}$ F. X. Bronold, A. Saxena, and A. R. Bishop, Phys. Rev. B 63, 235109 (2001).

${ }^{4}$ F. X. Bronold and H. Fehske, Phys. Rev. B 66, 073102 (2002).

${ }^{5}$ P. E. Kornilovitch, Phys. Rev. Lett. 81, 5382 (1998).

${ }^{6}$ J. P. Hague, P. E. Kornilovitch, J. H. Samson, and A. S. Alexan- drov, Phys. Rev. Lett. 98, 037002 (2007).

${ }^{7}$ A. S. Mishchenko and N. Nagaosa, in Polarons in Advanced Materials, Springer Series in Material Sciences Vol. 103, edited by A. S. Alexandrov (Springer, New York, 2007), p. 503.

${ }^{8}$ T. Holstein, Ann. Phys. (N.Y.) 8, 325 (1959); 8, 343 (1959). ${ }^{9}$ J. P. Hague, P. E. Kornilovitch, J. H. Samson, and A. S. Alexandrov, J. Phys.: Condens. Matter 19, 255214 (2007).

${ }^{10}$ A. S. Mishchenko, N. Nagaosa, A. Alvermann, H. Fehske, G. De Filippis, V. Cataudella, and O. P. Sushkov, arXiv:0806.2483 (unpublished). 